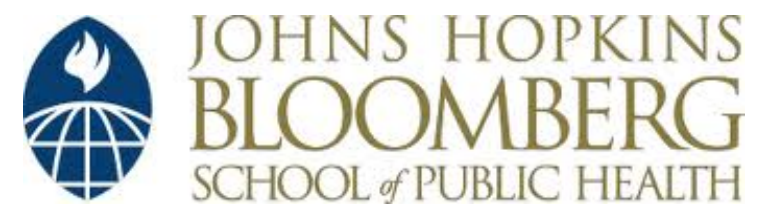

Johns Hopkins University, Dept. of Biostatistics Working Papers

$11-21-2003$

\title{
Underestimation of Standard Errors in Multi-Site Time Series Studies
}

\author{
Michael Daniels \\ Iowa State University,mdaniels@iastate.edu \\ Francesca Dominici \\ The Johns Hopkins Bloomberg School of Public Health, fdominic@jhsph.edu \\ Scott L. Zeger \\ The Johns Hopkins Bloomberg School of Public Health, szeger@jhsph.edu
}

\section{Suggested Citation}

Daniels, Michael ; Dominici, Francesca; and Zeger, Scott L., "Underestimation of Standard Errors in Multi-Site Time Series Studies" (November 2003). Johns Hopkins University, Dept. of Biostatistics Working Papers. Working Paper 13.

http://biostats.bepress.com/jhubiostat/paper13

This working paper is hosted by The Berkeley Electronic Press (bepress) and may not be commercially reproduced without the permission of the copyright holder.

Copyright $(\mathcal{C} 2011$ by the authors 


\title{
UNDERESTIMATION OF STANDARD ERRORS IN MULTI SITE TIME SERIES STUDIES
}

\begin{abstract}
Multi-site time series studies of air pollution and mortality and morbidity have figured prominently in the literature as comprehensive approaches for estimating acute effects of air pollution on health. Hierarchical models are generally used to combine site-specific information and estimate pooled air pollution effects taking into account both within-site statistical uncertainty, and across-site heterogeneity.
\end{abstract}

Within a site, characteristics of time series data of air pollution and health (small pollution effects, missing data, highly correlated predictors, non linear confounding etc.) make modelling all sources of uncertainty challenging. One potential consequence is underestimation of the statistical variance of the site-specific effects to be combined.

In this paper we investigate the impact of variance underestimation on the pooled relative rate estimate. We focus on two-stage normal-normal hierarchical models and on underestimation of the statistical variance at the first stage. By mathematical considerations and simulation studies, we found that variance underestimation does not affect the pooled estimate substantially. However, some sensitivity of the pooled estimate to variance underestimation is observed when the number of sites is small and underestimation is severe. These simulation results are applicable to any two-stage normal-normal hierarchical model for combining information of site-specific results, and they can be easily extended to more 
general hierarchical formulations.

We also examined the impact of variance underestimation on the national average relative rate estimate from the National Morbidity Mortality Air Pollution Study and we found that variance underestimation as much as $40 \%$ has little effect on the national average.

Key words: multi-site time series studies of air pollution and health, meta-analysis, hierarchical models, variance underestimation. 


\section{Introduction}

In multi-site time series studies of air pollution and mortality and morbidity $(1 ; 2)$, sitespecific time series data are assembled under a common framework and analyzed with a uniform analytic approach. Hierarchical modeling is an unified approach for combining evidence across studies, quantifying the sources of variability, and assessing effect modification. Because of the development of computational tools that facilitate their implementation $(3 ; 4)$, hierarchical models have been recently applied to analysis of multi-site time series data $(5 ; 1 ; 6 ; 7 ; 8 ; 9)$. See Dominici (2002) (10) for a more detailed discussion on the use of hierarchical models in multi-site time series studies of air pollution and health.

Hierarchical models (11) for analyses of multi-site time series studies of air pollution and mortality have a multi-stage structure. At the first stage, the association between air pollution and health is described using a site-specific regression model $(12 ; 13 ; 7)$ which takes into account potential confounding factors such as trend, season, and climate. Generalized Additive Models (GAM) (14) with non-parametric adjustment for confounding factors for the site (e.g. smoothing splines) or Generalized Linear Models (GLM)(15) with regression splines (e.g. natural cubic splines), are generally used for estimating site-specific relative rates $\hat{\beta}_{c}$ and their sampling variances $v_{c}$. Here $\hat{\beta}_{c}$ denotes the percentage increase in mortality/morbidity per unit increase in level of the air pollutant, and $v_{c}$ denotes the statistical uncertainty in $\hat{\beta}_{c}$ which depends on the number of: 1) days with available air pollution data; 2) adverse health events; and 3) correlated confounders. At the second stage, the information from multiple 
sites is combined by assuming that the true city-specific relative rates $\left(\beta_{c}\right)$ have a common mean $\alpha$ (also called the pooled relative rate) and variance $\tau^{2}$, which represents the variability across sites of the true relative rates (also called the heterogeneity parameter). Fixed or random effects models, empirical Bayesian, or fully Bayesian models $(16 ; 17 ; 18 ; 19 ; 20 ; 18)$ are used to estimate $\alpha$ and $\tau^{2}$.

The nature and characteristics of the time series studies in air pollution and health make estimation of health risk, taking into account of all sources of uncertainty, complex. First, the variability in the mortality time series explained by air pollution is an order of magnitude lower than the variability in the mortality time series explained by weather, trend and seasonality. Consequently the estimates of air pollution effects are sensitive to the method of adjustment for confounding factors. Second, to adequately control for confounding, several highly correlated predictors are included in the site-specific regression model. This can make variance estimation unstable and slow the convergence of fitting algorithms such as the backfitting algorithm in GAM (21). Third, because the confounding effects of climate and season are not linear, these needed to be modelled using smooth functions such as smoothing splines or regression splines (22). Non linear modelling increases the number of nuisance parameters and the computational complexity. In summary in all these cases, a sound and robust assessment of the statistical uncertainty of $\beta_{c}$ can be hard to obtain calling into questions the adequacy of $v_{c}$.

Problems inherent in standard errors estimation of air pollution effects have been recently pointed out in the literature. For example, Ramsey et al (2002) (23) reported that the in- 
ability of the GAM to properly take into account correlation among non linear confounders can lead to underestimation of the standard error of relative rate estimates (see also Chambers and Hastie (1992, pp 303-304) (24) and commentaries by Lumley and Sheppard (2003) and Samet et al. (2003) (25; 26).) The re-analyses of the National Morbidity Mortality Air Pollution Study (NMMAPS) (27) empirically confirm theoretical results of Ramsey et al. (2002) (23), and show that the degree of bias in the standard errors is proportional to the size of the standard errors (a form of multiplicative bias). More robust variances than the ones obtained from GAM software can be obtained by using standard statistical theory (28), by bootstrap (29) or by GEE methods (30). However in time series studies of air pollution and health, such methods might be computationally expensive and 'off-the-shelf' statistical software is not always available.

In this paper, we investigate the sensitivity of the pooled estimate $\alpha$ with respect to underestimation of the city-specific statistical variances $v_{c}$. We focus on the underestimation problem for three reasons. First, underestimation of $v_{c}$ is a much more serious problem than overestimation because it leads to less conservative conclusions about statistical significance of a site-specific association between air pollution and health. Second, underestimation of $v_{c}$ is more common than overestimation because the former generally reflects failing to take into account one or more sources of uncertainty. Third, underestimation of $v_{c}$ has been identified as a limitation of the statistical software for the implementation of GAM $(24 ; 23)$.

We show that the pooled estimate is unaffected by underestimation of $v_{c}$ when: 1) the bias is additive, and 2) the bias is multiplicative and the statistical variances are equal across 
cities. Then by a simulation study, we investigate the case of multiplicative bias and unequal statistical variances. Here we define $3^{3}=27$ scenarios which are a combination of: 1 ) number of cities; 2) magnitude of the bias; and 3) amount of heterogeneity, and identify under which scenarios the underestimation of $v_{c}$ affects the estimate of the overall parameter $\alpha$. We also investigate the impact of variance underestimation on the national average relative rate estimate from the National Morbidity Mortality Air Pollution Study (NMMAPS).

\section{Methods and Results}

We consider the following two stage normal-normal hierarchical model (11)

$$
\begin{aligned}
& \hat{\beta}_{c}=\beta_{c}+N\left(0, v_{c}\right), c=1, \ldots, C \\
& \beta_{c}=\alpha+N\left(0, \tau^{2}\right)
\end{aligned}
$$

where $C$ is the total number of sites, and $N(a, b)$ denotes the normal distribution with mean $a$ and variance $b$.

We estimate $\alpha$ and it standard error by using an Empirical Bayes approach (EB)(18). More specifically, we first compute the Restricted Maximum Likelihood Estimate (REML) $\hat{\tau}^{2}$, and then we estimate $\alpha$ and its standard error conditional on $\hat{\tau}^{2}$. Details are in the appendix. The EB estimate of $\alpha$ and its standard error are defined below:

$$
\begin{aligned}
\hat{\alpha} & =\sum_{c} \hat{\beta}_{c} / w_{c} / \sum_{c} 1 / w_{c} \\
S E(\hat{\alpha}) & =1 / \sqrt{\sum_{c} 1 / w_{c}} .
\end{aligned}
$$

where $w_{c}=v_{c}+\hat{\tau}^{2}$. We consider three cases of underestimation of $v_{c}$ : 
1. additive bias: $v_{c}^{\star}=v_{c}-b$, for arbitrary $v_{c}$ and $b>0$;

2. multiplicative bias, equal variances: $v_{c}^{\star}=k \times v_{c}, v_{c} \equiv v$ and $k<1$;

3. multiplicative bias, unequal variances: $v_{c}^{\star}=k \times v_{c}$, for arbitrary $v_{c}$ and $k<1$.

In the first and second case, underestimation of the variance does not affect the pooled estimate of $\alpha$. The mathematical proof is detailed in the appendix.

In the third case, we investigate the impact of multiplicative bias on the estimation of $\alpha$ by a simulation study. We consider the following $3^{3}=27$ scenarios:

- underestimation of $50 \%, 30 \%$ and $10 \%$ which corresponds to $k=0.5,0.7,0.9$;

- number of sites: $C=15,20,90$;

- amount of heterogeneity: $\tau=0.05,0.5,1$ corresponding, respectively, to small, medium, and large between city standard deviations.

For each scenario, we generated $250 \hat{\beta}_{c}$ s from model (1). For $C=90$, we set $v_{c}$ equal to the estimates obtained from the NMMAPS re-analysis (27). For $C=15$ and $C=20$ we take a random sample from the $90 v_{c}$ NMMAPS estimates. We also set $\alpha$ equal to 0.21 (the pooled NMMAPS estimate for total mortality at lag $1(27)$ ). In summary, each scenario (biased $v_{c}^{\star}$, sample size $C$, and amount of heterogeneity $\tau$ ), leads to 250 simulated values of $\hat{\beta}_{c}$.

For each simulated $\hat{\beta}_{c}$ and for each scenario, we calculate the empirical Bayes estimates of $\tau^{2}$ and $\alpha$, using both $v_{c}$ and with $v_{c}^{\star}$, by fitting model (1) and using equation (2). This 
leads to two sets of 250 estimates of $\alpha$. Figure 1 shows boxplots of the 250 standardized differences between the two estimates of $\alpha$. Each standardized difference is obtained by taking the difference between the two estimates of $\alpha$ and by dividing it by the standard deviation of the 250 estimates of $\alpha$ under model (1) with $v_{c}$. This transforms each difference into standard deviation units, e.g a difference larger than 2 indicates statistically significant difference between the two estimates of $\alpha$, or in other words, strong indication of sensitivity of the $\alpha$ estimate to the use of the biased $v_{c}^{\star}$ instead of $v_{c}$.

For all scenarios, the $95 \%$ confidence intervals of the standardized differences was always within 2 standard deviations of 0 , suggesting that underestimation of $v_{c}$ does not affect $\hat{\alpha}$ substantially. In eight scenarios, the distributions of the standardized differences show more variability with their maximum absolute differences larger than 2 standard deviations. Seven of those scenarios corresponded to extreme underestimation of $50 \%$. The other scenario was characterized by small sample size, small heterogeneity, and a bias of $30 \%$.

We also investigate the impact of variance underestimation on the NMMAPS national average relative rate estimate. We re-calculate the national average relative rate of mortality for 10 units increases in $P M_{10}$ by varying the underestimation parameter $k$ from 0.1 ( $90 \%$ variance underestimation) to 1 (no variance underestimation). Figure 2 shows the estimates of the national average, $\hat{\alpha}$, their standard errors $S E(\hat{\alpha})$, and the heterogeneity $\hat{\tau}$ as function of the underestimation parameter $k$. Note that for values of $k$ increasing from 0.1 to 0.6 (variance underestimation from 90 to 40 percent), $\hat{\tau}$ decreases, and $\hat{\alpha}$ moves from the un-weighted average of the $\hat{\beta}_{c}$ toward the weighted average of the $\hat{\beta}_{c}$ defined in equation (2). 
The latter occurs due to the fact that when $k$ is very small (large variance underestimation), $\hat{\tau}$ is large relative to $v_{c}^{*}=k v_{c}$, and roughly the same weight is assigned to the city-specific estimates. In addition, over the same range of $\mathrm{k}(0.1 \leq k \leq 0.6), S E(\hat{\alpha})$ decreases because: 1) $S E(\hat{\alpha})$ is an increasing function of $\hat{\tau}$ for fixed $v_{c}^{\star}$ (see equation 2), and 2) $\hat{\tau}^{2}$ decreases more quickly with $k$ than $v_{c}^{*}=k v_{c}$. For values of $k$ increasing from 0.6 to 1 (variance underestimation from 40 to 0 percent), $\hat{\alpha}$ is constant because $\hat{\tau}^{2}=0$, and $S E(\hat{\alpha})$ increases since $v_{c}^{\star}=k v_{c}$ increases.

In summary, little or no effect is observed when the variance underestimation is less than $40 \%(k \geq 0.6)$ leading to a national average estimate equal to $0.21 \%$ and standard error 0.05 . When underestimation is larger than $40 \%(k<0.6)$, then $\hat{\alpha}$ gradually increases with the degree of underestimation toward the un-weighted pooled estimate (which is approximately equal to $0.28 \%$ ). However, for all values of $k$, the t-ratio $\hat{\alpha} / S E(\hat{\alpha})$ remains larger than 2 indicating statistical significance of the national average relative rate estimate.

\section{Discussion}

The results of this paper indicate that in multi-site time series studies of air pollution and health, underestimation of the statistical variances $v_{c}$ does not affect the estimate of the pooled effect $\alpha$ substantially. Some sensitivity is observed when the number of sites is small (less than 20), the between city variability is close to zero, and the underestimation is larger than $40 \%$. 
This robustness only applies to the estimation of the pooled effect $\alpha$. EB estimates of the site-specific relative rates $\beta_{c}, \tilde{\beta}_{c}$, are indeed affected by the underestimation of the $v_{c}$. To see this, consider their definition:

$$
\tilde{\beta}_{c}=\hat{\beta}_{c} S_{c}+\hat{\alpha}\left(1-S_{c}\right)
$$

where $S_{c}=v_{c}^{-1} /\left(\hat{\tau}^{-2}+v_{c}^{-1}\right)$ is the shrinkage factor. Note that $S_{c}$ is not invariant to underestimation of the $v_{c}$. In this case, underestimation of $v_{c}$ leads to overestimation of $S_{c}$ and therefore leads to $\tilde{\beta}_{c}$, which rely too heavily on $\hat{\beta}_{c}$. In summary, variance underestimation leads to an overestimation of the heterogeneity of the air pollution effects, and therefore to under-shrinkage of the city-specific EB estimates toward their overall mean $\hat{\alpha}$.

Unfortunately because the true statistical variances are unknown, the distinction between additive versus multiplicative bias is not straightforward. One possibility is to specify two alternative but comparable site-specific regression models and compare the estimates of $v_{c}$ under the two models. For example Dominici et al. (2002) estimated $\hat{\beta}_{c}$ and $v_{c}$ under a GLM with natural cubic splines and under a GAM with smoothing splines and compared the $v_{c}$ under these two modelling approaches. Alternatively, a more robust estimate of the statistical variance can be performed for a small number of cities and compared with the available ones. More robust estimate of $v_{c}$ are always encouraged and they can be obtained by using: 1) asymptotic theory and calculating the inverse of the information matrix (28); 2) bootstrap (29); 3) or sandwich estimates (30). 
Results in this paper are presented using an empirical Bayesian approach to estimation, where the Bayesian estimate of $\alpha$ is obtained by plugging in a point estimate of $\tau^{2}$ in Equation 2 . However our results apply also if a fully Bayesian version of the above model are fit with non-informative priors on $\tau^{2}$ and $\alpha$.

Finally, the results of this paper apply under the assumption that the normal approximation to the likelihood function at the first stage of the hierarchical model is appropriate. Asymptotically, this approximation has an accuracy proportional to the number of days with available data in each city (31). In time series studies of air pollution and health the asymptotic normal approximation is generally accurate, however additional work is needed to extend such results to distributions other than the normal and to examine the sensitivity of inferences if the normal approximation is not accurate.

\section{Appendix}

Details on the estimation of $\tau^{2}$ and $\alpha$. The EB estimate of $\alpha$ is obtained by first computing the REML of $\tau^{2}$, which is then plugged in the equation (2). The REML of $\tau^{2}$ is obtained by maximizing the following likelihood function

$$
\operatorname{Lik}\left(\tau^{2} \mid \hat{\beta}_{c}, v_{c}\right)=\left(\sum_{c} w_{c}\left(\tau^{2}\right)^{-1}\right)^{-1 / 2} \prod_{c=1}^{C} w_{c}\left(\tau^{2}\right)^{-1 / 2} \exp \left\{-\frac{1}{2} \sum_{c=1}^{C} w_{c}\left(\tau^{2}\right)^{-1}\left[\hat{\beta}_{c}-\hat{\alpha}\left(\tau^{2}\right)\right]^{2}\right\}
$$


where $\hat{\alpha}\left(\tau^{2}\right)=\left[\sum_{c} \hat{\beta}_{c} w_{c}\left(\tau^{2}\right)^{-1}\right] /\left[\sum_{c} w_{c}\left(\tau^{2}\right)^{-1}\right]$ and $w_{c}\left(\tau^{2}\right)=v_{c}+\tau^{2}$.

\section{Mathematical arguments concerning the robustness of the pooled estimate to variance underestimation under cases 1 and 2 .}

Under scenario 1 , we start by showing that additive bias does not affect estimation of $\alpha$ for arbitrary $v_{c}$. Let's $\tau^{2 \star}$ be the maximum likelihood estimate (mle) of $\tau^{2}$ obtained by maximizing the likelihood (3) with $v_{c}^{\star}$ instead of $v_{c}$. Note that estimation of $\alpha$ defined in equation (2) depends on $v_{c}$ only through $v_{c}+\tau^{2}$. Therefore we simply need to show that the estimate for $\tau^{2 \star}$ conditional on $v_{c}^{\star}$ is $b$ units more than the estimate for $\tau^{2}$ conditional on $v_{c}$. In this case $v_{c}+\hat{\tau}^{2}=v_{c}^{\star}+\hat{\tau}^{2 \star}$ and therefore $\hat{\alpha}\left(\hat{\tau}^{2}\right)$ is unaffected by underestimation of $v_{c}$. We define $\tau^{2 \star}=\tau^{2}+b$ and we maximize the likelihood (3) (with $v_{c}=v_{c}^{\star}$ ) with respect to $\tau^{2 \star}$. By the invariance property of the maximum likelihood estimates, if $\hat{\tau}^{2 \star}$ is mle then $\hat{\tau}^{2}$ is also mle. Therefore $v^{c}+\hat{\tau}^{2}=\left(v^{c}-b\right)+\left(\hat{\tau}^{2}+b\right)=v_{c}^{\star}+\hat{\tau}^{2 \star}, \hat{\tau}^{2^{\star}}=\hat{\tau}^{2}+b$.

Under scenario 2, we assume multiplicative bias with equal variances $v_{c}=v$. Here $v_{c}^{\star}=k \times v_{c}$ can be re-written as $v_{c}^{\star}=v_{c}-(1-k) \times v_{c}$. Because $v_{c}=v$, then $(1-k) \times v_{c}=(1-k) \times v=b$ and therefore $v_{c}^{\star}=v_{c}-b$. So the results above for additive bias apply here also. 


\section{References}

[1] Katsouyanni K, Toulomi G, Spix C, et al. Short term effects of ambient sulphur dioxide and particulate matter on mortality in 12 european cities: results from time series data from the APHEA project. British Medical Journal, 314:1658-1663, 1997.

[2] Samet JM, Zeger SL, Dominici F, Dockery D, Schwartz J. The National Morbidity, Mortality, and Air Pollution Study Part I: Methods and Methodological Issues. Health Effects Institute, Cambridge, MA., 2000.

[3] Thomas A, Spiegelhalter DJ, Gilks WR. Bugs: A program to perform Bayesian inference using Gibbs sampling. In Bayesian Statistics 4. Proceedings of the Fourth Valencia International Meeting, 837-842. Clarendon Press: Oxford, 1992.

[4] Gilks WR, Richardson S, Spiegelhalter DJ, eds. Markov Chain Monte Carlo in Practice. Chapman and Hall, London, 1996.

[5] Burnett R, Krewski D. Air pollution effects of hospital admission rates: A random effects modelling approach. The Canadian Journal of Statistics, 22:441-458, 1994.

[6] Roemer W, Hoek G, Brunekreef B, Kalandidi A, Pekkanen J. Daily variations in air pollution and respiratory health in a multicenter study: the peace project. European Respiratory Journal, 12:1354-1361, 1998.

[7] Dominici F, Samet JM, Zeger SL. Combining evidence on air pollution and daily mortal- 
ity from the twenty largest US cities: A hierarchical modeling strategy (with discussion). Royal Statistical Society Series A with discussion, 163:263-302, 2000.

[8] Zanobetti A, Schwartz J, Dockery D. Airborne particles are a risk factor for hospital admissions for heart and lung disease. Environmental Health Perspective, 108:10711077, 2000.

[9] Schwartz J. Assessing confounding, effect modification, and thresholds in the associations between ambient particles and daily deaths. Environmental Health Perspective, 108:563-568, 2000.

[10] Dominici F. Air pollution and health: what can we learn from an hierarchical approach? invited commentary. American Journal of Epidemiology, 1:11-15, 2002.

[11] Lindley DV, Smith AFM. Bayes estimates for the linear model (with discussion). Journal of the Royal Statistical Society Series B Methodological, 34:1-41, 1972.

[12] Samet JM, Zeger SL, Berhane K. The Association of Mortality and Particulate Air Pollution. Health Effects Institute, Cambridge, MA., 1995.

[13] Kelsall J, Samet JM, Zeger SL. Air pollution, and mortality in Philadelphia, 1974-1988. American Journal of Epidemiology, 146:750-762, 1997.

[14] Hastie TJ, Tibshirani RJ. Generalized additive models. Chapman and Hall, New York, 1990. 
[15] McCullagh P, Nelder JA. Generalized Linear Models (Second Edition). New York: Chapman \& Hall, 1989.

[16] Hedges LV, Olkin I. Statistical Methods for Meta-analysis. Academic Press, 1985.

[17] DerSimonian R, Laird N. Meta-analysis in clinical trials. Controlled Clinical Trials, 7:177-188, 1986.

[18] Carlin BP, Louis TA. Bayes and Empirical Bayes Methods for Data Analysis. Chapman \& Hall, New York, 1996.

[19] Morris CN, Normand SL. Hierarchical models for combining information and for metaanalysis. In JM Bernardo, JO Berger, AP Dawid, AFM Smith, eds., Bayesian Statistics 4, 321-344, Oxford, 1992. Oxford University Press.

[20] Gelman A, Carlin J, Stern H, Rubin D. Bayesian Data Analysis. Chapman and Hall, London, 1995.

[21] Buja A, Hastie T, Tibshirani R. Linear smoothers and additive models. The Annals of Statistics, 17:453-455, 1989.

[22] Green PJ, Silverman BW. Nonparametric Regression and Generalized Linear Models: A Roughness Penalty Approach. Chapman \& Hall, London UK, 1994.

[23] Ramsay T, Burnett R, Krewski D. The effect of concurvity in generalized additive models linking mortality and ambient air pollution. Epidemiology to appear, 2002.

[24] Chambers J, Hastie T. Statistical Models in S. Chapman and Hall, London, 1992. 
[25] Lumley T, Sheppard L. Time series analyses of air pollution and health: Straining at gnats and swallowing camels? Epidemiology, 14:?-?, 2003.

[26] Samet J, Dominici F, McDermott A, Zeger S. New problems for an old design: Time series analyses of air pollution and health. Epidemiology, 14:?-?, 2003.

[27] Dominici F, McDermott A, Zeger SL, Samet JM. A Report to the Health Effects Institute on Reanalyses of the NMMAPS Database. The Health Effects Institute, Cambridge, MA., 2002.

[28] van der Vaart AW. Asymptotic Statistics. Cambridge Series in Statistics and Probalistic Mathematics, Cambridge University Press, 1998.

[29] Efron B, Tibshirani RJ. An Introduction to the Bootstrap. Chapman \& Hall, New York, 1993.

[30] Liang KY, Zeger SL. Longitudinal data analysis using generalized linear models. Biometrika, 73:13-22, 1986.

[31] Daniels M, Kass R. A note on first-stage approximation in two-stage hierarchical models. Sankhya Series B, 60:19-30, 1998. 


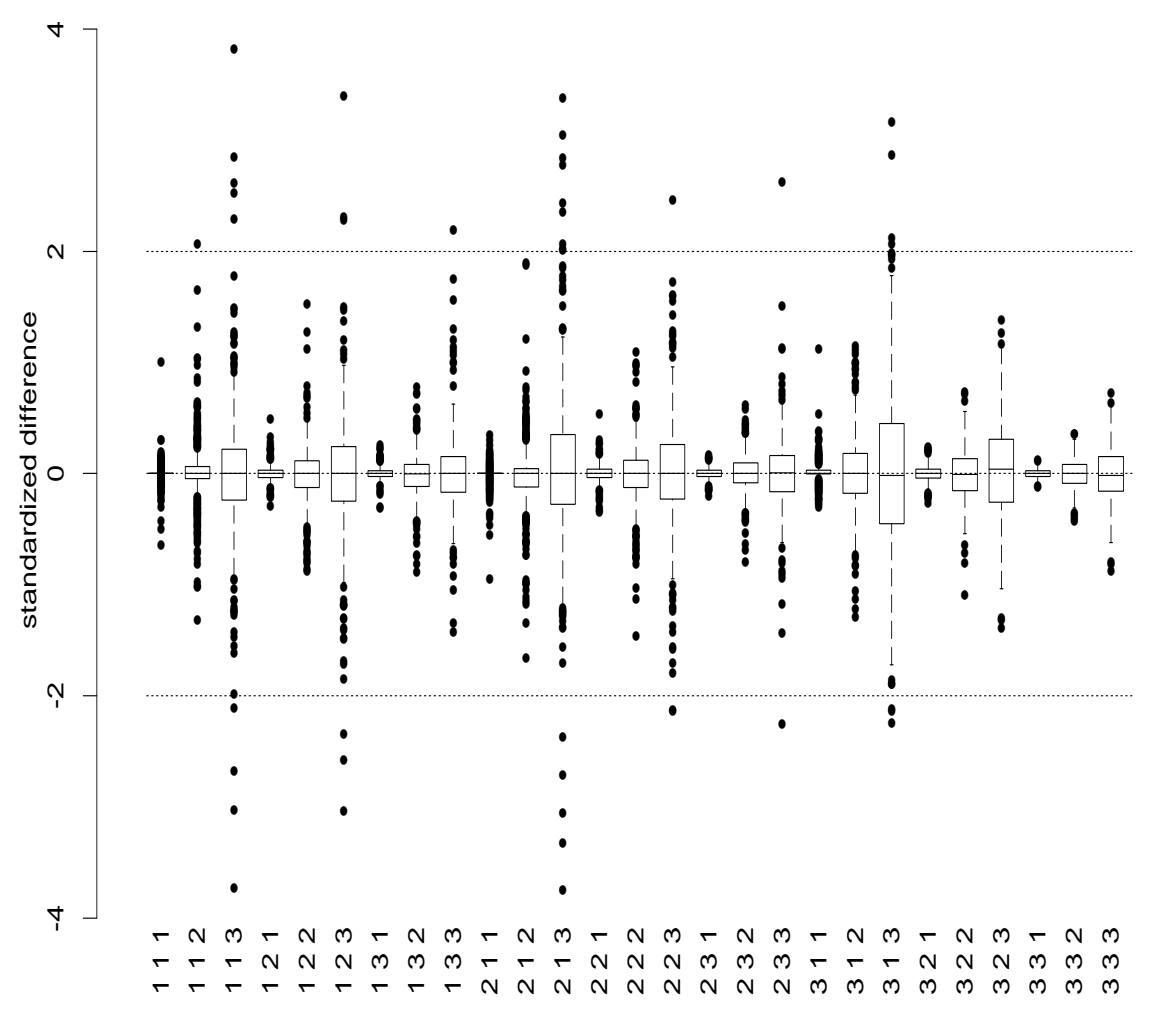

Figure 1: Boxplots of the standardized differences between the $\alpha$ 's estimates under model (1) with $v_{c}$ and $v_{c}^{\star}$. The three digit labels, $(i, j, l)$ on the x-axis, correspond to the simulation scenarios. The first digit corresponds to the sample size $(i=1: C=15 ; i=2: C=20$; and $i=3: C=90)$. The second digit corresponds to the heterogeneity parameter $(j=$ $1: \tau=0.05 ; j=2: \tau=0.5$; and $j=3: \tau=1.0)$. The third digit corresponds to the underestimation parameter $(l=1: k=0.10 ; l=2: k=0.30$; and $l=3: k=0.50)$. 

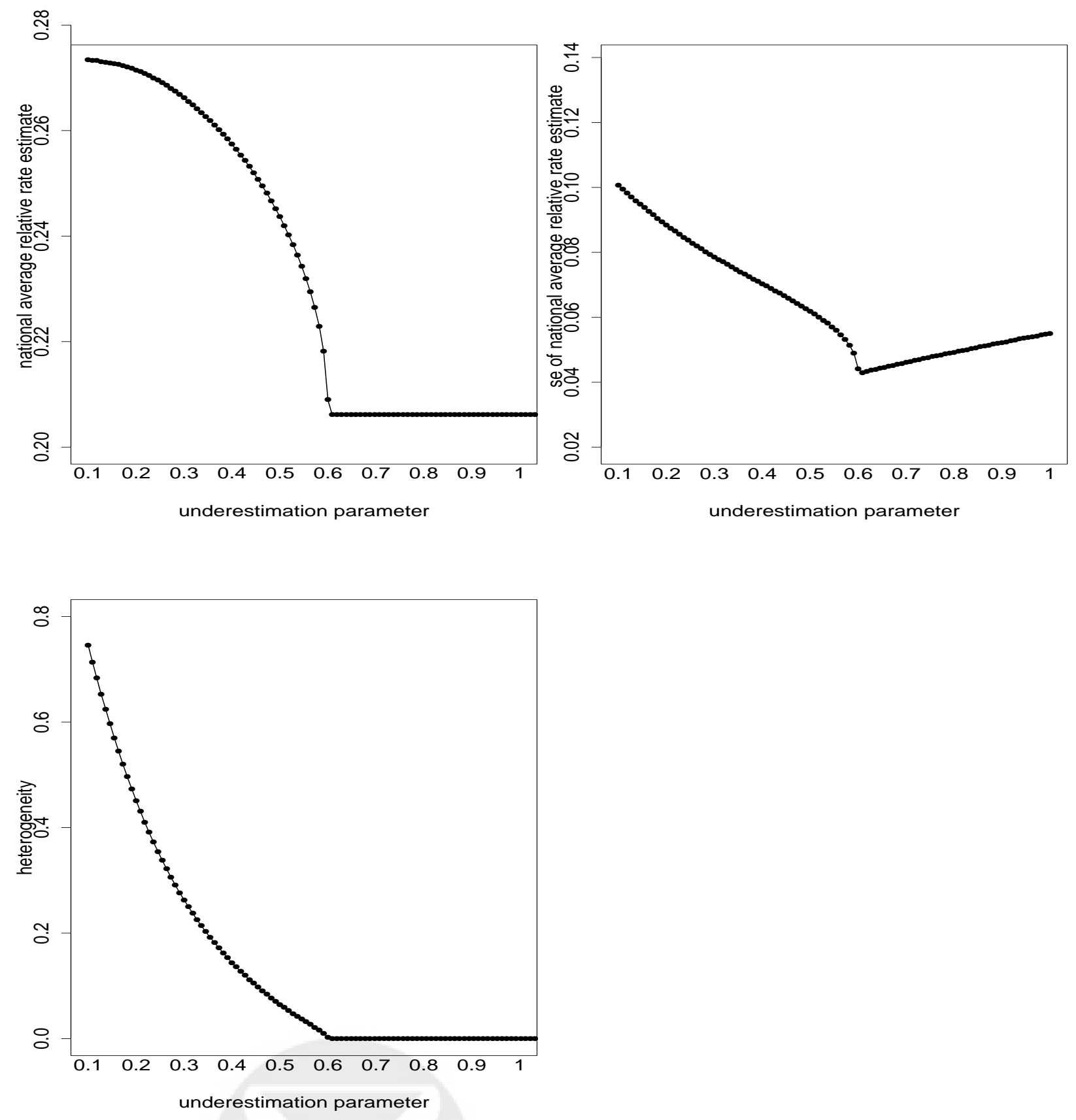

Figure 2: Plots of $\hat{\alpha}, S E(\hat{\alpha})$, and heterogeneity parameter $\hat{\tau}$ as function of the underestimation parameter, $k$ for the NMMAPS data. 